\title{
The millimeter-wave bolometric interferometer
}

\author{
Andrei L. Korotkov ${ }^{* a}$, Jaiseung Kim ${ }^{\text {a }}$, Gregory S. Tucker ${ }^{\mathrm{a}}$ \\ Amanda Gault ${ }^{\mathrm{b}}$, Peter Hyland ${ }^{\mathrm{b}}$, Siddharth Malu, Peter T. Timbie ${ }^{\mathrm{b}}$ \\ Emory F. Bunn ${ }^{\mathrm{c}}$, Evan Bierman ${ }^{\mathrm{d}}$, Brian Keating ${ }^{\mathrm{d}}$, Anthony Murphy ${ }^{\mathrm{e}}$, Créidhe O'Sullivan $^{\mathrm{e}}$ \\ Peter A. R. Ade ${ }^{\mathrm{f}}$, Carolina Calderon ${ }^{\mathrm{f}}$, Lucio Piccirillo ${ }^{\mathrm{f}}$ \\ ${ }^{\mathrm{a}}$ Dept. of Physics, Box 1843, Brown Univ., 182 Hope St., Providence, RI, USA 02912; \\ ${ }^{\mathrm{b}}$ Dept. of Physics, Univ. of Wisconsin, 1150 University Ave., Madison, WI USA 53706 \\ ${ }^{c}$ Dept. of Physics, Univ. of Richmond, 28 Westhampton Way, Richmond, VA USA 23173 \\ ${ }^{\mathrm{d}}$ Dept. of Physics, Univ. of California, San Diego, 9500 Gilman Drive, La Jolla, CA USA 92093 \\ ${ }^{\mathrm{e}}$ Dept. of Exp. Physics, National Univ. of Ireland, Maynooth, Co. Kildare, Ireland \\ ${ }^{\mathrm{f}}$ Dept. of Physics and Astronomy, Univ. of Wales, Queen's Building 5 The Parade, Cardiff, CF24 \\ 3YB United Kingdom
}

\begin{abstract}
The Millimeter-Wave Bolometric Interferometer (MBI) is designed for sensitive measurements of the polarization of the cosmic microwave background (CMB). MBI combines the differencing capabilities of an interferometer with the high sensitivity of bolometers at millimeter wavelengths. It views the sky directly through corrugated horn antennas with low sidelobes and nearly symmetric beam patterns to avoid spurious instrumental polarization from reflective optics. The design of the first version of the instrument with four 7-degree-FOV corrugated horns (MBI-4) is discussed. The MBI-4 optical band is defined by filters with a central frequency of $90 \mathrm{GHz}$. The set of baselines determined by the antenna separation makes the instrument sensitive to CMB polarization fluctuations over the multipole range $l=150-270$. In MBI4, the signals from antennas are combined with a Fizeau beam combiner and interference fringes are detected by an array of spider-web bolometers with NTD germanium thermistors. In order to separate the visibility signals from the total power detected by each bolometer, the phase of the signal from each antenna is modulated by a ferrite-based waveguide phase shifter. Observations are planned from the Pine Bluff Observatory outside Madison, WI.
\end{abstract}

Keywords: CMB polarization, interferometer, bolometer.

\section{INTRODUCTION}

We are in the process of building an initial version of an instrument called the Millimeter-wave Bolometric Interferometer for observing faint astrophysical sources. The main scientific goal of MBI is to search for primordial $B-$ mode polarization in the cosmic microwave background (CMB).

A significant challenge in $\mathrm{CMB}$ polarization measurements is separation of the very weak pseudoscalar $B$ component from the much stronger scalar $E$ component ${ }^{1,2}$. The two types of polarization modes probe different physical phenomena; in particular, ordinary density (scalar) perturbations produce only $E$-type polarization (to linear order). As a result, the $B$ component is predicted to be smaller than $E$ by an order of magnitude or more over all angular scales. However, the very fact that density perturbations do not produce $B$-type polarization makes detection of the $B$ component more valuable: the $B$ channel is a clean probe of other types of perturbations.

The most exciting prospect is the use of $B$ modes to detect primordial gravitational waves (tensor perturbations) produced during an inflationary epoch. If this tensor $B$ component is detected, we will have a direct probe of the Universe at far earlier times than any other method can provide. The other dominant source of $B$-type polarization in the $\mathrm{CMB}$ is expected to be gravitational lensing of $E$ modes by large-scale structure. These two predicted sources of $B$ modes probe very different epochs: the tensor contribution is imprinted on the CMB at the time of last scattering but is a relic of the extremely early Universe; the lensing contribution is produced at much later times. The lensing signal peaks at relatively small angular scales $\left(<1^{\circ}\right)$, while the tensor signal (if it exists) is strongest on large scales.

*andrei_korotkov@physics.brown.edu; phone 1401 863-1466; fax 1401 863-2024

Millimeter and Submillimeter Detectors and Instrumentation for Astronomy III, edited by Jonas Zmuidzinas, Wayne S. Holland, Stafford Withington, William D. Duncan, Proc. of SPIE Vol. 6275, 62750X, (2006) · 0277-786X/06/\$15 - doi: 10.1117/12.672246

Proc. of SPIE Vol. $627562750 X-1$ 
Some of the most powerful instruments for observing the CMB and diffuse emission at millimeter and sub-millimeter wavelengths are interferometers. The Sunyaev-Zel'dovich effect has been imaged by the Ryle ${ }^{3}$, OVRO, BIMA and SZA interferometers ${ }^{4,5,6}$ at centimeter wavelengths. The CMB has been imaged by the $\mathrm{CAT}^{7}, \mathrm{DASI}^{8}, \mathrm{CBI}^{9,10}$ and $\mathrm{VSA}^{11}$ interferometers, also at centimeter wavelengths. DASI was the first instrument to detect the CMB polarization ${ }^{12,13}$. All of these instruments use coherent receivers based on HEMT amplifiers.

MBI is the first, to our knowledge, application of interferometry using incoherent detectors (bolometers) at millimeter wavelengths ${ }^{14}$. Cooled bolometers are the highest-sensitivity broadband detectors at millimeter and sub-millimeter wavelengths; in low-background applications they can achieve the fundamental noise limit imposed by photon noise from the CMB itself. MBI combines the differencing capabilities of interferometry with the high sensitivity of bolometers and results in a new instrument with capabilities that would be difficult to achieve using more traditional techniques. Unlike a single-dish imaging telescope, an interferometer instantaneously performs a differential measurement: the effective "beam pattern" of each individual baseline is a set of fringes that sample the sky with positive and negative weights. This differencing removes the need for mechanical chopping or rapid scanning. Only correlated signals are detected, so the interferometer has reduced sensitivity to changes in the total power signal absorbed by the detectors ${ }^{15}$. Thus the signal from an interferometer is significantly less affected by the atmosphere ${ }^{16,17}$. Unless a full-sky map (an impossibility because of Galactic cuts) is made with infinite angular resolution the $E$ and $B$ modes "leak" into each other ${ }^{18,19}$. The sampling of each baseline is very narrow in Fourier space, which is ideal for power spectrum measurements and $E-B$ separation $^{20}$. There are other advantages achieved in combining the two technologies:

- A simple optical design, combined with interferometry, reduces systematic effects.

- All of the optics is at a temperature of $4 \mathrm{~K}$ or below.

- Scaling to a large number of elements involves replicating simple components.

- The technique is scalable to all wavelengths needed for foreground removal $(\sim 30-300 \mathrm{GHz})$.

The initial version of the MBI instrument, called MBI-4, is nearly complete. MBI-4 has four interferometer apertures and uses sixteen bolometers; it has an instantaneous field of view of $\sim 7^{\circ}$ and a resolution of $\sim 1^{\circ}$. MBI-4 is a single frequency-band (with $90 \mathrm{GHz}$ central frequency) instrument designed as a pathfinder for future CMB polarization measurements such as NASA's $\mathrm{CMBPol}^{21}$ mission; its sensitivity is adequate for detecting CMB temperature anisotropy and for conducting a range of tests to characterize system performance and systematic effects.

\section{INSTRUMENT}

\subsection{The adding interferometer}

In a simple 2-element radio interferometer, signals from two antennas aimed at the same point in the sky are correlated so that the sky temperature is sampled with an interference pattern with a single spatial frequency. The output of such an interferometer is the visibility, $V(\vec{u}) \propto \int G(\hat{x}) \Delta T(\hat{x}) e^{i 2 \pi u \cdot \hat{x}} d \hat{x}$, where $\hat{x}$ is a unit 3-vector in the direction of a point on the sky, $G(\hat{x})$ is the beam pattern of each antenna (assumed to be identical), and $T(\hat{x})$ is the map of the temperature fluctuations on the sky we are trying to measure. $\left(T(\hat{x}) \propto E_{0}^{2}\right.$.) The vector $\vec{u}$ has length $B / \lambda$, where $B$ is the baseline length, and is oriented along the baseline. With more antennas these same correlations are performed along each baseline. To recover the full phase information, complex correlators are used to measure simultaneously both the in-phase and quadrature phase components of the visibility.

In interferometers that use incoherent detectors, such as MBI, the electric field wavefronts from two telescopes are added and then squared in a detector as shown in Fig. 1 - an "adding" interferometer as opposed to a "multiplying" interferometer ${ }^{22}$. The result is a constant term proportional to the intensity plus an interference term. The constant term is an offset that is removed by phase modulating one of the signals. Phase-sensitive detection at the modulation frequency recovers the interference term and reduces susceptibility to low-frequency drifts ( $1 / f$ noise) in the bolometer and readout electronics. The adding interferometer recovers the same visibility as a multiplying interferometer.

The polarization of radiation is completely characterized by the Stokes parameters, $U, Q, V$, and $I$. If the incoming wave is expressed as $\vec{E}=E_{x} \hat{i}+E_{y} \hat{j} e^{i \phi}$, then the linear polarization Stokes parameters are $U=\left\langle 2 E_{x} E_{y} \cos \phi\right\rangle$ and 
$Q=\left\langle E_{x}^{2}-E_{y}^{2}\right\rangle$, the circular polarization parameter is $V=\left\langle 2 E_{x} E_{y} \sin \phi\right\rangle$, and the intensity $I=\left\langle E_{x}^{2}+E_{y}^{2}\right\rangle$. The brackets denote a time average. Stokes $Q$ and $U$ are related to each other by a rotation: an instrument that measures $U$ can be rotated by $45^{\circ}$ to measure Stokes $Q$, and vice-versa.

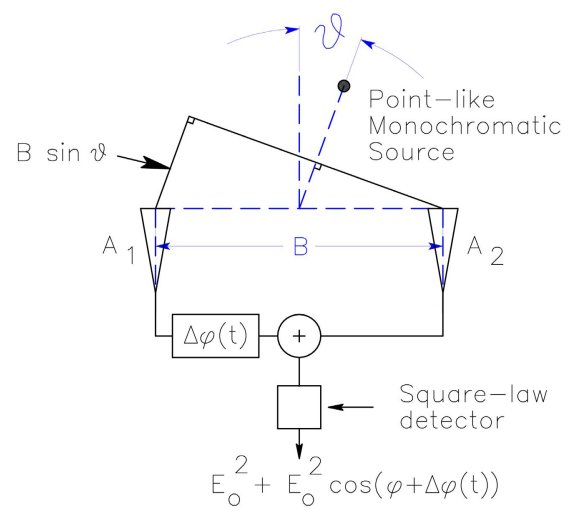

Fig. 1. Adding interferometer. At antenna $A_{2}$ the electric field is $E_{0}$, and at $A_{1}$ it is $E_{0} e^{i \phi}$, where $\phi=k B \sin \theta$ and $k=2 \pi / \lambda . B$ is the length of the baseline, and $\theta$ is the angle of the source with respect to the symmetry axis of the baseline, as shown. (For simplicity consider only one wavelength, $\lambda$, and ignore time dependent factors.) In a multiplying interferometer the in-phase output of the correlator is proportional to $E_{0}^{2} \cos \phi$. For the adding interferometer, the output is proportional to $E_{0}^{2}+E_{0}^{2} \cos (\phi+\Delta \phi(t))$. The desired signal is recovered by introducing a time dependent phase modulation $\Delta \phi(t)$ in one arm of the interferometer

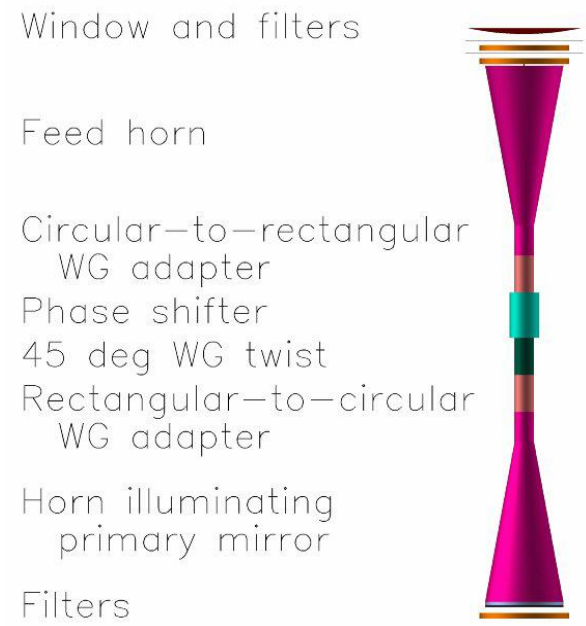

Fig. 2. Input unit (IU) of the MBI-4 interferometer. All optical components are housed in a cryostat. With the exception of the cryostat window and input filters all of the optical components are at $4 \mathrm{~K}$. Light enters from the top and passes through three sets of filters at $300 \mathrm{~K}, 77 \mathrm{~K}$, and $4 \mathrm{~K}$ before entering the feed horn. The IUs sensitive to orthogonal polarizations have left- and right-handed $45^{\circ}$ waveguide (WG) twists, so the outgoing polarization vectors are aligned. A $\pm 90^{\circ}$ phase modulation can be introduced by the ferrite-based phase shifter. WGs and the system of thermal and blocking filters provide the $20 \%$ bandwidth with $90 \mathrm{GHz}$ central frequency. In a future MBI instrument, an ortho-mode transducer will be inserted into each IU after the feed horn, so each IU will have two phase shifters, two illuminating horns, and be sensitive to two orthogonal polarizations.

An interferometer measures the Stokes parameters directly, without differencing the signal from separate detectors. It correlates the components of the electric field captured by each antenna with the components from all of the other antennas. On the baseline formed by two antennas, 1 and 2, the interferometer's correlators measure $\left\langle E_{1 x} E_{2 x}\right\rangle$, $\left\langle E_{1 y} E_{2 y}\right\rangle,\left\langle E_{1 x} E_{2 y}\right\rangle$, and $\left\langle E_{1 y} E_{2 x}\right\rangle$. The first two are used to determine $I$ and the latter two measure $U$. Rotating the instrument allows a measurement of $Q$. (Stokes $V$ can be recovered in a similar manner but is expected to be zero for the CMB; we do not plan on measuring it.) To measure these values with a single baseline, the antennas must be sensitive to both polarizations with further separation by ortho-mode transducer (OMT). MBI-4 only collects a single polarization for each feed (Fig. 2), so does not use an OMT. Two of the four feeds (see Fig. 3) are sensitive to one polarization of the incoming radiation, and the other two to the orthogonal mode. Four of the baselines are sensitive to polarization (Stokes $U$ ), while the remaining two are sensitive to temperature fluctuations (Stokes $I$ ). The instrument will rotate in $10^{\circ}$ steps around its optical axis to provide reasonable coverage of the $u-v$ plane (see Fig. 4).

The sensitivity of a receiver to broadband signals increases as the square root of the bandwidth. For interferometers, the bandwidth restricts the angular range, $\theta$, over which fringes are detected ${ }^{23,24}$. If we assume the path lengths for a source at the center of the FOV are equal, then the path length difference for a source at an angle $\theta$ from the center along the baseline axis is $\theta B$, where $B$ is the baseline distance. If this path length difference is small compared to the coherence length of the light, $\lambda^{2} / \Delta \lambda$, then the fringe contrast is not affected. Thus the FOV is determined by 
$\theta_{F O V} \leq(\lambda / \Delta \lambda)(\lambda / B)$. This equation indicates that for angles of the order of the product of the spectral resolution times the angular resolution, the fringe smearing is important. This relation imposes restrictions on the ratio between the maximum baseline achievable by the interferometer and the spectral bandwidth of the receiver. For MBI-4, the bandwidth is $20 \%$, which sets the maximum baseline to about four times the diameter of each antenna.

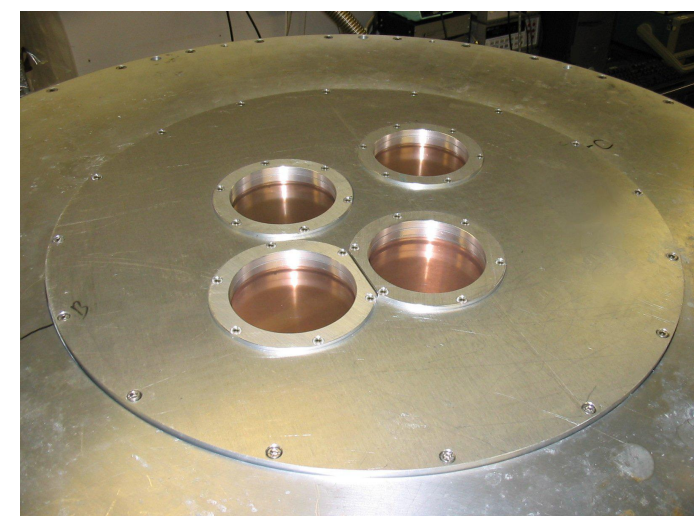

Fig. 3. The input of MBI-4. The input filters in front of the input corrugated conical feed horns are visible through the transparent vacuum windows. Each of the four channels is sensitive to a single polarization of the incoming field: "diagonal" elements are sensitive to the same polarization, while "neighboring" elements are sensitive to orthogonal polarizations. The windows can be replaced with Zote-foam.

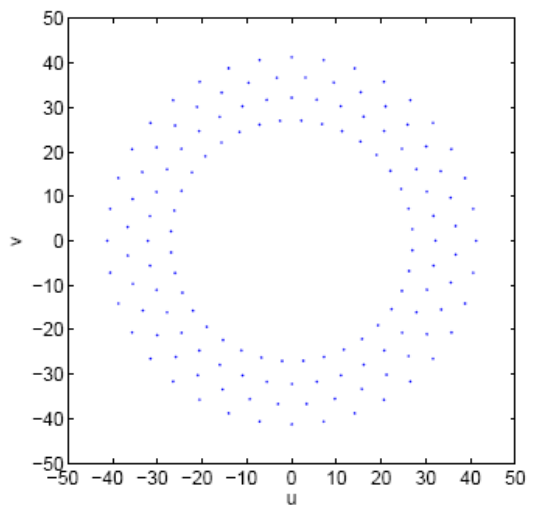

Fig. 4. The "polarization sensitive" $u-v$ coverage of MBI-4 for the four $90 \mathrm{GHz}$ channels. The instrument is rotated in 10 steps about its optical axis during observations. This makes the instrument sensitive to $\mathrm{CMB}$ polarization fluctuations over the multipole range $l=150-270$.

\subsection{Control of systematic effects}

The $E$-mode CMB polarization signal is small, and the $B$-mode polarization signal is smaller. The $B$-mode signal will be at most $\sim 0.1 \mu \mathrm{K}$, so control and minimization of systematic effects is essential.

$\mathrm{Hu}$ et al. ${ }^{25}$ have studied systematics in imaging polarization experiments, and we have extended their analysis to interferometers. The temperature anisotropy is large compared to any polarization signal; therefore, systematic errors that convert temperature anisotropy into an apparent polarization anisotropy (Stokes $I$ into $Q$ and $U$ ) are particularly dangerous. Interferometers differ from single-dish imaging telescopes in which systematic effects lead to such mixing. For instance, in an interferometer there is no conversion from $I$ into $Q$ and $U$ from gain errors, differential pointing errors, or beam mismatches between different antennas.

MBI simultaneously measures both temperature anisotropy and polarization. The polarization channels are designed to be insensitive to $T$, i.e., to have a high common-mode rejection.

Corrugated feedhorns have extremely low sidelobes and have easily calculable, symmetric beam patterns. Furthermore, there are no reflections from optical surfaces to induce spurious instrumental polarization, an unavoidable problem for any system with imaging optics ${ }^{26,27,28,29}$. In MBI there are no aberrations from off-axis pixels: all feed elements are equivalent and all the optics are at cryogenic temperatures to minimize losses. Note that any off-axis optics in the MBI system occur after the phase modulators, so do not produce a modulated polarization signal.

As an interferometer MBI is inherently insensitive to beam ellipticity. In a future version of MBI, we may wish to operate the instrument in a correlation radiometer mode as well as an interferometer. In this case, beam ellipticity is, in principle, a source of systematic error; however, the very symmetric patterns of the corrugated conical feed horns minimize this effect as well ${ }^{30}$. The rotation of MBI about its optical axis also reduces this effect.

\subsection{Sensitivity}

In order to separate the interference terms from each other we modulate the phase of the antenna signals in a sequence. By differencing the detector outputs that appear during each phase state, we can isolate the various visibilities. This scheme amounts to a kind of time-domain multiplexing of the signals from the various baselines onto each detector. 
The expected sensitivity of MBI was estimated by following the approach developed by Zmuidzinas ${ }^{31}$. The sensitivity of an interferometer is comparable to that of a filled-dish telescope collecting the same number of modes from the sky.

\subsection{Galactic foregrounds}

A key goal of any CMB polarization experiment must be to separate the polarized signals emitted at the era of decoupling from those emitted much more recently by our own Galaxy. A better understanding of the spectral and spatial characteristics of the Galactic foregrounds is needed so that we can devise better methods (e.g. "templates") for effecting this separation.

We have chosen to operate MBI at wavelengths where the dominant foreground will be dust ${ }^{32}$. Dust grains align with the Galactic magnetic field ${ }^{33}$, so we can probe Galactic fields. These fields are well coupled to the interstellar medium (ISM) and have energy density comparable to the other major components of the ISM. For these reasons, they play important roles in the ISM, including confining cosmic rays, moderating interstellar shocks, propagating turbulence, and influencing star formation.

Observations of the thermal component of the dust radiation have revealed that the wavelength- dependence of the polarization is surprisingly strong. The observations are few, and are specifically limited to molecular cloud envelopes, but they give a consistent picture of a degree of polarization that falls with wavelength in the far-infrared, and then begins to rise in the submillimeter, near $350 \mu \mathrm{m}$ wavelength. These results have been explained using a model in which the efficiency of grain alignment is correlated with exposure to radiation from both embedded stars and external sources $^{34,35}$. The importance of understanding the physics of grain alignment lies principally in the fact that studies of polarized dust emission provide one of the few methods for mapping magnetic fields, especially in the dense regions where stars form. Without the physical understanding it is difficult to determine, for any given line-of-sight, which specific regions are being sampled within an extended, heterogeneous and complex molecular cloud.

\section{TECHNICAL APPROACH}

\subsection{Corrugated feed horns}

The observation of the sky directly with feed horns has several advantages. The optical design is simple and clean. A large number of feed horns, not limited in number by a telescope design, can be used to obtain sensitivity. The cost of this approach is the loss of angular resolution unless extremely large feed horns are used. MBI uses this approach, but adds interferometry between feed horns to recover some of the angular resolution lost by dispensing with a telescope.

In MBI-4 we have used electroformed corrugated conical feed horns for the input elements. These feed horns have a symmetric beam pattern with a measured beam FWHM of $\sim 7^{\circ}$. MBI-4 only collects a single polarization for each feed selected by the rectangular WR-10 waveguide attached to the horn output. In a future MBI instrument a waveguide ortho-mode transducer ${ }^{36}$ will be used.

The relative placement of the feed horn is chosen in order to provide uniform $u-v$ coverage for polarization sensitive channels with $10^{\circ}$ step rotation of the instrument around its optical axis (Fig. 4). This set of baselines makes the instrument sensitive to CMB polarization fluctuations over the multipole range $1=150-270$. "Temperature" channels will be used for calibration by comparison with temperature maps of WMAP.

\subsection{Phase modulator}

In order to separate the interference (visibility) signals from the total power signal detected by each bolometer, the phase of the signal from each antenna must be modulated. The phase is sequentially modulated between $-90^{\circ}$ and $+90^{\circ}$.

For MBI-4 we are using ferrite-based phase modulators ${ }^{37}$; these waveguide devices are a modification of the Faraday rotators used in BiCEP. The modulation rate is $\sim 10-100 \mathrm{~Hz}$. The loss in the phase shifter is $<1 \mathrm{~dB}$. The magnetic field in the ferrite is controlled by a small superconducting coil. The phase shifters dissipate negligible power, $\sim 1 \mathrm{~mW}$ each. Also, the differential loss between the two phase states must be small. Differential loss will produce an "offset" signal after demodulation of the detector signal.

\subsection{Beam combiner}

The signals from each of the input units (IUs) are interfered using a so-called Fizeau ${ }^{38}$ beam combiner. The Fizeau combiner acts as an image-plane correlator or interferometer. In our instrument, the Fizeau combiner is essentially a 
Cassegrain telescope. All signals from the IUs illuminate the primary mirror, and the light is correlated or interfered on the array of 16 bolometers at the focal plane behind the primary mirror.

\subsection{Detectors, electronics and data acquisition}

MBI-4 uses 16 traditional spider-web bolometers, provided by JPL, with NTD germanium thermistors. The bolometers are placed in an optical cavity (see Fig. 5) and coupled to the incoming radiation via conical horns. The horns form a hexagonally packed array with spacing $2.8 \mathrm{~cm}$ in the image-plane of the beam combiner. The whole unit is suspended from the supporting frame by Kevlar threads and connected to the cold plate of the ${ }^{3} \mathrm{He}$ refrigerator. The optical efficiency for this configuration is expected to be $\sim 50 \%$.

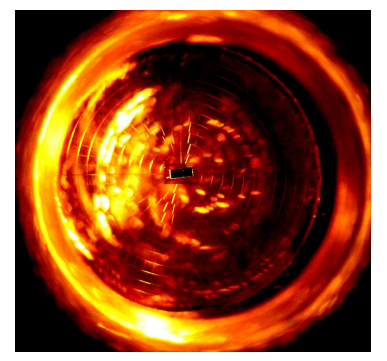

Fig. 5. A spider-web JPL bolometer, with NTD germanium thermistor. The detectors are coupled to the incoming radiation via conical horns. The entire bolometer unit with 16 detectors is at $330 \mathrm{mK}$.

The MBI-4 bolometers are read out with a standard AC-biased differential circuit. The readout circuit demodulates the detector signals to provide stability to low frequencies $(<30 \mathrm{mHz})$. The bolometer bias and readout electronics are based on those of BLAST ${ }^{39}$. The preamplifiers consist of Siliconix U401 differential JFETs with $5-7 \mathrm{nV} / \mathrm{Hz}^{1 / 2}$ noise at $v>100 \mathrm{~Hz}$ and $120 \mu \mathrm{W}$ power dissipation per pair. They are suspended on a lithographed silicon nitride membrane, using fabrication techniques similar to those used to make the bolometers and self-heat to the optimal operating temperature of $120 \mathrm{~K}$. The total power of the JFETs for 16 channels is only $4 \mathrm{~mW}$, which allows them to be placed close to the detectors.

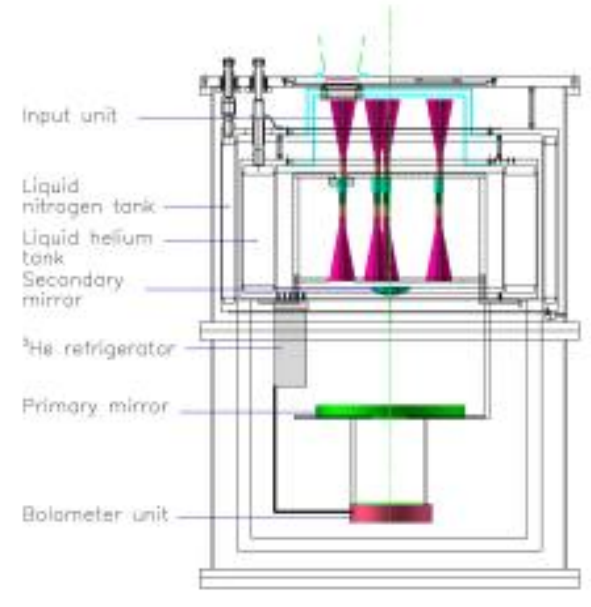

Fig. 6. The MBI-4 optics and cryostat. The four IUs are visible at the top of the cryostat. The bolometer unit, cooled by the ${ }^{3} \mathrm{He}$ refrigerator, also contains filters. The cryostat uses both liquid nitrogen and liquid helium.

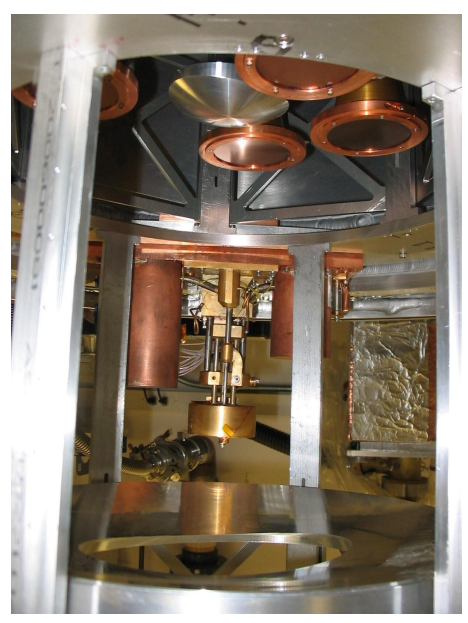

Fig. 7. MBI-4. At the top are the four IUs and secondary mirror of the quasi-optical Fizeau beam combiner. At the bottom is the primary mirror of the beam combiner. The bolometers are located below the primary mirror and are not visible. Visible at the back is the ${ }^{3} \mathrm{He}$ refrigerator.

\subsection{Cryogenics}

A schematic of the MBI-4 instrument is shown in Fig. 6 and a photograph of the receiver is shown in Fig. 7. The cryostat holds 17 liters of liquid nitrogen and 25.7 liters of liquid helium. In its operational configuration the liquid helium lasts 
for 50 hours. The detectors are cooled by a self-contained ${ }^{3} \mathrm{He}$ refrigerator manufactured by Simon Chase. The ${ }^{3} \mathrm{He}$ condenser is cooled by a self-contained charcoal-pumped ${ }^{4} \mathrm{He}$ pot. The base temperature of the ${ }^{3} \mathrm{He}$ refrigerator in its operational configuration is $330 \mathrm{mK}$ and lasts at least 90 hours. Cycling the refrigerator takes about one hour. The refrigerator is designed so that an additional ${ }^{3} \mathrm{He}$ stage can be attached to the first ${ }^{3} \mathrm{He}$ stage, which would provide lower temperatures $(\sim 200 \mathrm{mK})$.

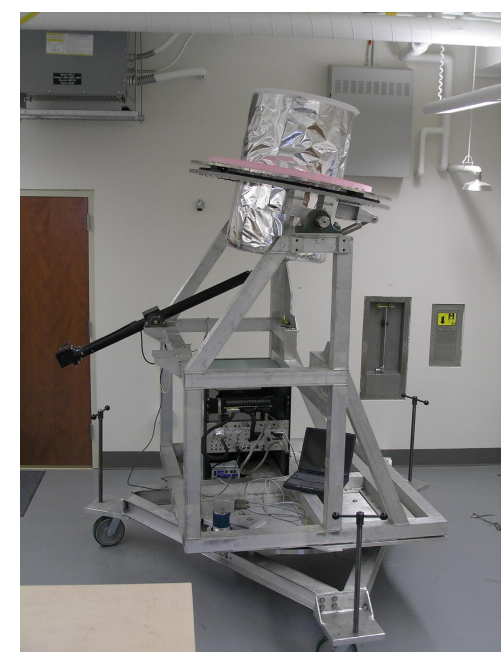

Fig. 8. The MBI-4 pointing system. The azimuth is driven by two hardened metal disks in pressure contact; a small disk is directly driven by a motor which causes a large diameter disk, and hence the azimuth axis, to turn. The elevation axis is driven by a ball screw. Visible at the top of the mount is the bearing which allows the cryostat to rotate about the optical axis. (In this photograph, the actual cryostat is not installed.) Near the bottom of the mount is the control and signal conditioning electronics.

\subsection{Telescope and mount}

The MBI pointing platform, shown in Fig. 8, consists of a fully-steerable altitude-azimuth mount. In addition, the entire cryostat can be rotated around the optical ("theta") axis. Tracking of the sky occurs under computer control using feedback from 17-bit absolute optical encoders on each of the three axes - altitude, azimuth and theta. Absolute pointing is established using a bore-sited optical telescope. This altitude - azimuth mounting scheme was used successfully on the COMPASS experiment ${ }^{41}$.

\section{FUTURE PLANS}

We will demonstrate MBI-4 at the Pine Bluff Observatory (PBO) near Madison, Wisconsin. Key tests include measuring the interferometric beam patterns, observing bright object such as the moon, and during the winter, when atmospheric conditions are good, carrying out long integrations on test fields.

We are studying how the design of MBI can be optimized for future measurements of the CMB. We envision scaling MBI-4 to form modules that include 16 horn antennas and that correlate both polarizations from each antenna. These modules could be scaled to operate at a range of millimeter wavelengths. Multiple modules at each of a variety of wavelengths would allow removal of foreground sources and would increase sensitivity to the levels required for CMB polarization observations.

\section{ACKNOWLEDGMENTS}

This work is supported by NASA grant NAG5-12758, Rhode Island Space Grant, and Wisconsin Space Grant. 


\section{REFERENCES}

1. Zaldarriaga M. and Seljak, U., "All-sky analysis of polarization in the microwave background," $P R D, 55,1830$ (1997)

2. M. Kamionkowski, A. Kosowsky and A. Stebbins, "Statistics of cosmic microwave background polarization," $P R D$, 55,7368 (1997)

3. M. Birkinshaw, "The Sunyaev-Zel'dovich effect," Phys. Rept., 310, 97 (1999)

4. J. E. Carlstrom, M. Joy, and L. Grego, "Interferometric Imaging of the Sunyaev-Zeldovich Effect at $30 \mathrm{GHz}$," ApJ, 456, L75 (1996)

5. J. E. Carlstrom, M. Joy, and L. Grego, "Interferometric Imaging of the Sunyaev-Zeldovich Effect at $30 \mathrm{GHz}$ : Erratum," ApJ, 461, L59 (1996)

6. S. J. C. Muchovej, J. E. Carlstrom, J. K. Cartwright, C. Greer, D. Hawkins, R. Hennessy, M. Joy, J. Lamb, E. Leitch, M. Loh, A. Miller, T. Mroczkowski, C. Pryke, B. Reddall, G. Richardson, M. Runyan, M. Sharp, and D. Woody, "First Results from the Sunyaev-Zel'dovich Array," Bulletin of the AAS, 207.4102M (2005)

7. P. F. Scott, R. Saunders, G. Pooley, C. O'Sullivan, A. N. Lasenby, M. Jones, M. P. Hobson, P. J. Duffett-Smith, and J. Baker, "Measurements of Structure in the Cosmic Background Radiation with the Cambridge Cosmic Anisotropy Telescope," ApJ, 461, L1 (1996)

8. N. W. Halverson, J. E. Carlstrom, M. Dragovan, W. L. Holzapfel, and J. Kovac, "DASI: Degree Angular Scale Interferometer for imaging anisotropy in the cosmic microwave background," Proc. SPIE, 3357, 416 (1998)

9. J. K. Cartwright, T. J. Pearson, A. C. S. Readhead, M. C. Shepherd, and J. L. Sievers, "Limits on the polarization of the cosmic microwave background radiation at multipole up to $1 \sim 2000$, , ApJ, 623, 11 (2005)

10. S. Padin, M. C. Shepherd, J. K. Cartwright, R. G. Keeney, B. S. Mason, T. J. Pearson, A. C. S. Readhead, W. A. Schaal, J. Sievers, P. S. Udomprasert, J. K. Yamasaki, W. L. Holzapfel, J. E. Carlstrom, M. Joy, S. T. Myers, and A. Otarola, "The Cosmic Background Imager," PASP, 114, 83 (2002)

11. C. Dickinson, et al., "High sensitivity measurements of the CMB power spectrum with the extended Very Small Array," MNRAS 353, 732 (2004)

12. E. M. Leitch, J. M. Kovac, N W. Halverson, J. E. Carlstrom, C. Pryke, and M. W. E. Smith, "Degree Angular Scale Interferometer 3 Year Cosmic Microwave Background Polarization Results," ApJ, 624, 10 (2005)

13. E. M. Leitch, J. M. Kovac, C. Pryke, B. Reddall, E. S. Sandberg, M. Dragovan, J. E. Carlstrom, N. W. Halverson, and W. L. Holzapfel, "Measuring Polarization with DASI," Nature, 420, 763 (2002)

14. G. S. Tucker, J. Kim, P. Timbie, S. Ali, L. Piccirillo, and C. Calderon, "Bolometric interferometry: the millimeterwave bolometric interferometer," New Astronomy Reviews, 47, 1173 (2003)

15. M. White, J. E. Carlstrom, M. Dragovan, and W. L. Holzapfel, Interferometric Observation of Cosmic Microwave Background Anisotropies," ApJ, 514, 12 (1999)

16. S. E. Church, "Predicting residual levels of atmospheric sky noise in ground-based observations of the Cosmic Background Radiation," MNRAS, 272, 551 (1995)

17. O. P. Lay and N. W. Halverson, "The Impact of Atmospheric Fluctuations on Degree-Scale Imaging of the Cosmic Microwave Background," ApJ, 543, 787 (2001)

18. A. Lewis, A. Challinor, and N. Turok, "Analysis of CMB polarization on an incomplete sky," PRD, 65, 023505 (2002)

19. E. F. Bunn, M. Zaldarriaga, M. Tegmark and A. de Oliveira-Costa, "E/B decomposition of finite pixelized CMB maps," PRD, 67, 023501 (2003)

20. C. G. Park and K.-W. Ng, "E/B Separation in Cosmic Microwave Background Interferometry," ApJ, 609, 15 (2004)

21. R. Weiss, et al., "Task Force on Cosmic Microwave Background Research: Final Report," (2005) http://www.nsf.gov/mps/ast/tfcr_nal report.pdf

22. K. Rohlfs, and T. L. Wilson, Tools of Radio Astronomy, 2nd ed., Springer, New York (1996)

23. A. R. Thompson, J. M. Moran, and G. W. Swenson Jr., "Interferometry and Synthesis in Radio Astronomy," Krieger Publishing Company, Malabar, Florida (1998)

24. T. Boker and , J. A. Allen, "Imaging and Nulling with the Space Interferometer Mission," ApJS, 125, 123 (1999)

25. W. Hu, M. M. Hedman, M. Zaldarriaga, "Benchmark parameters for CMB polarization experiments," PRD, D67, $043004(2003)$

26. C. Rosset, "Systematic E_ects in CMB Polarization Measurements," Conference XXXIXth Rencontres de Moriond, "Exploring the Universe," La Thuile (2005) 
27. S. Mitra, A. S. Sengupta, and T. Souradeep, "CMB power spectrum estimation using noncircular beams," PRD, 70, $103002(2004)$

28. E. Carretti, S. Cortiglioni, C. Sbarra, and R. Tascone, "Antenna instrumental polarization and its effects on E- and B-modes for CMBP observations," A\&A, 420, 437 (2004)

29. E. Carretti, R. Tascone, S. Cortiglioni, J. Monari, and M. Orsini, "Limits due to instrumental polarisation in CMB experiments at microwave wavelengths," New Astronomy, 6, 173 (2001)

30. J. Kaplan and J. Delabrouille, "Some sources of systematic errors on CMB polarized measurements with bolometers," Astrophysical Polarized Backgrounds: Workshop on Astrophysical Polarized Backgrounds, Cecchini, S., Cortiglioni, S., Sault, R. \& Sbarra, C., eds., AIP Conf. Proc., 609, 209 (2002)

31. Zmuidzinas, J., "Cramêr-Rao sensitivity limits for astronomical instruments: implications for interferometer design,” J. Opt. Soc. America A Optics, Image Science and Vision, 20, 218 (2003)

32. L. Page, G. Hinshaw, E. Komatsu, M. R. Nolta, D. N. Spergel, C. L. Bennett, C. Barnes, R. Bean, O. Doré, M. Halpern, R. S. Hill, N. Jarosik, A. Kogut, M. Limon, S. S. Meyer, N. Odegard, H. V. Peiris, G. S. Tucker, L. Verde, J. L. Weiland, E. Wollack, and E. L. Wright, "Three Year Wilkinson Microwave Anisotropy Probe (WMAP) Observations: Polarization Analysis," submitted to ApJ (2006)

33. A. Lazarian and S. Prunet, "Polarized Microwave Emission from Dust," in AIP Conf. Proc. 609: Astrophysical Polarized Backgrounds, AIP, Melville, NY (2002)

34. J. E. Vaillancourt, "Analysis of far-infrared/submillimeter polarization spectrum based on temperature maps of Orion," ApJS, 142, 53 (2002)

35. R. H. Hildebrand, J. L. Dotson, C. D. Dowell, D. A. Schleuning, and J. E. Vaillancourt, "The Far-Infrared Polarization Spectrum: First Results and Analysis," ApJ 516, 834 (1999)

36. E. J. Wollack, "A Full Waveguide Band Orthomode Junction," Electronics Division Internal Report, National Radio Astronomy Observatory, Green Bank, $W V$, No. 303, May 1996

37. B. G. Keating, J. J. Bock, N. Erickson, T. Gaier, A. E. Lange, J. Rorie, D. Siegal, and E. Wollack, "Linear Polarization Modulator for mm-Wavelengths," in preparation (2006)

38. H. Fizeau, C. R. Acad. Sci. Paris, 66, 932 (1868)

39. G. S. Tucker, P. A. R. Ade, J. J. Bock, M. Devlin, M. Griffin, J. Gundersen, M. Halpern, P. Hargrave, D. Hughes, J. Klein, C. B. Netterfield, L. Olmi, and D. Scott, "The Balloon-borne Large Aperture Submillimeter Telescope (BLAST)," Advances in Space Research, 33, 1793 (2004)

40. E. J. Wollack and S. H. Moseley, "Corrugated platelet feed arrays for millimeter wave imaging," National Radio Science Meeting, International Union of Radio Science, Boulder, CO (2005)

41. P. C. Farese, G. Dall'Oglio, J. O. Gundersen, B. G. Keating, S. Klawikowski, L. Knox, A. Levy, P. M. Lubin, C. W. O'Dell, A. Peel, L. Piccirillo, J. Ruhl, and P. T. Timbie, "COMPASS: An Upper Limit on Cosmic Microwave Background Polarization at an Angular Scale of 20,", ApJ 610, 625 (2004) 